\title{
F-SAR - Recent Upgrades and Campaign Activities
}

\author{
Ralf Horn, Marc Jaeger, Martin Keller, Markus Limbach, Anton Nottensteiner, Matteo \\ Pardini, Andreas Reigber, Rolf Scheiber
}

\author{
German Aerospace Center DLR e.V., Microwaves and Radar Institute \\ Muenchner Strasse, 20, Wessling, GERMANY \\ Email: ralf.horn@dlr.de
}

\begin{abstract}
The F-SAR instrument represents DLR's advanced airborne SAR testbed for technology and remote sensing applications. The development of the instrument was triggered by a strong demand for data being simultaneously acquired at different wavelengths and polarizations as well as by the demand for very high range resolution. F-SAR is a modular development utilizing modern hardware and commercial of the shelf components. For the purpose of experiments and operational data acquisition campaigns the system is being installed on board a DLR Dornier DO228 research aircraft.

This paper gives an overview of the instrument's capabilities and performance, based on the multi-frequency and fully polarimetric imagery acquired during campaigns in the last two years. More specifically, campaigns in Greenland in 2015 (ARCTIC) and Gabon in 2016 (AfriSAR) are presented.
\end{abstract}

\section{F-SAR Instrument Overview}

F-SAR operates fully polarimetric at X-, C-, S-, L- and P-bands and features single-pass polarimetric interferometric SAR (PolInSAR) capabilities in X- and S-bands. Repeat-pass Pol-InSAR (RP-PolInSAR) is a measurement mode available for all bands. Range resolution is determined by available signal bandwidth. While components and ITU regulations limit the signal bandwidth to $50 \mathrm{MHz}$ at P-band, a step-frequency approach is adopted to achieve up to $760 \mathrm{MHz}$ of effective signal bandwidth at X-band to satisfy the requirement for very high resolution [1]. A summary of the general technical parameters can be found in Tab. 1.

Table 1. Technical parameters of the F-SAR instrument.

\begin{tabular}{llllll}
\hline & X & C & S & L & P \\
\hline RF [GHz] & 9.60 & 5.30 & 3.25 & 1.325 & 0.435 \\
Bandwidth [MHz] & 760 & 384 & 300 & 150 & 50 \\
PRF [kHz] & 5 & 5 & 5 & 10 & 10 \\
PT [kWpeak] & 2.50 & 2.20 & 2.20 & 0.90 & 0.90 \\
Duty factor [\%] & 5 & 5 & 5 & 10 & 10 \\
Rg. resolution [m] & 0.3 & 0.6 & 0.75 & 1.5 & 2.25 \\
Az. resolution [m] & 0.2 & 0.3 & 0.35 & 0.4 & 1.5 \\
Flight altitude range & From 2000ft above ground level to 22000ft above mean sea level (msl) tested \\
Off-nadir angle range & Nominal from 25 to 60 & & & \\
Ground range coverage & From 600m to 6km proportional to flight altitude & \\
Operational endurance & According to system configuration between 2.5h and 5h & \\
Sampling & 8 Bit real; 1GS/500MS selectable; max number of samples 64k per range line; \\
four recording channels & & \\
Data rates & Up to 190MByte/s (per rec. channel) & \\
\hline
\end{tabular}

The 18th International Radar Symposium IRS 2017, June 28-30, 2017, Prague, Czech Republic 978-3-7369-9343-3 (C2017 DGON 
All radar subsystems underwent intensive testing in the field, meaning real environment, to optimise their performance. This includes, of course, the dedicated off-line signal processing facility, the F-SAR Processor.

F-SAR is a right-looking SAR, meaning all antennas of all bands are pointing to the right hand side of the aircraft. Figure 1 shows an artist's view of how the radar operates simultaneously in four frequency bands in a single overflight.
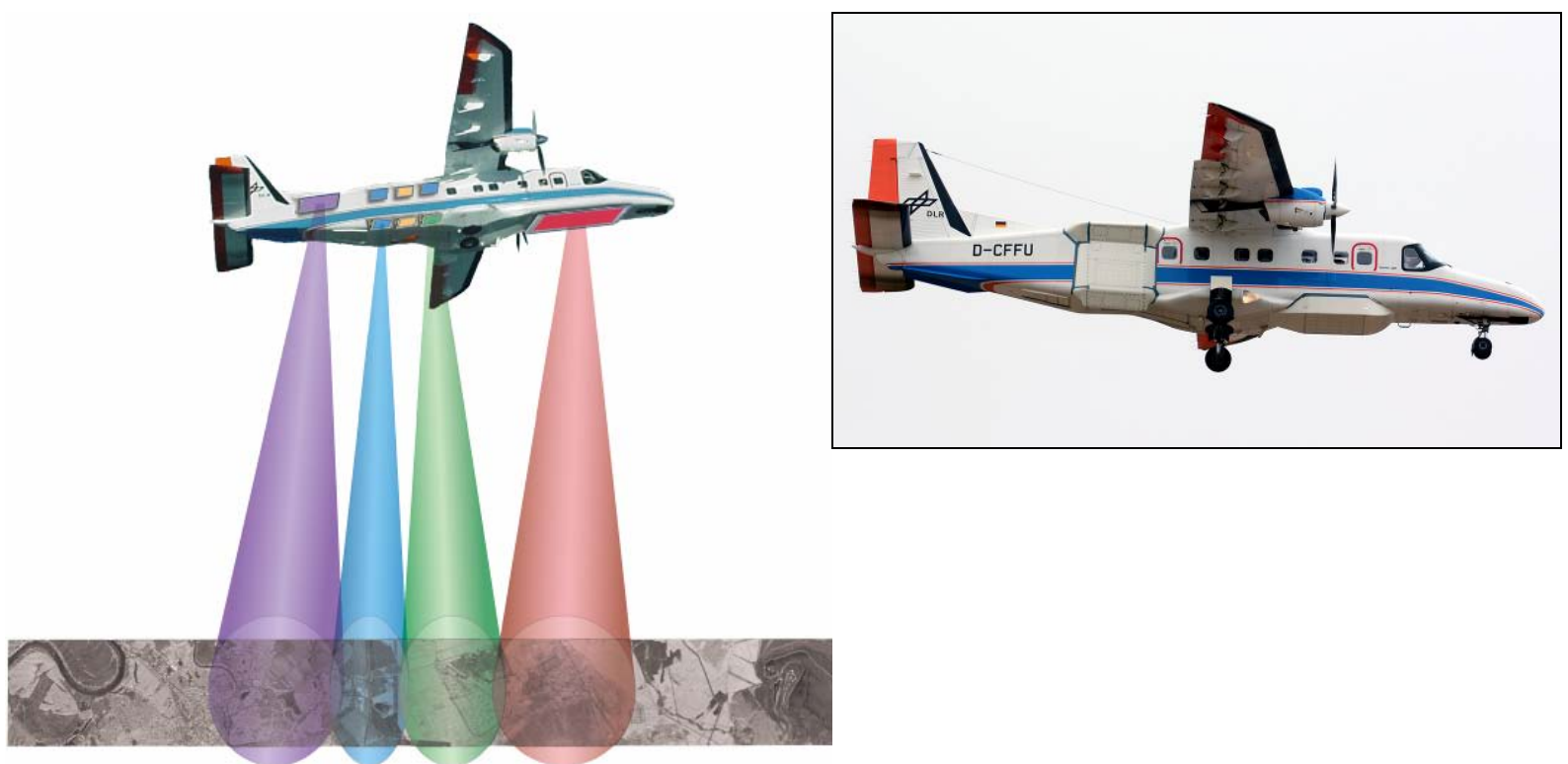

Figure 1. F-SAR radar on board DLR DO228 acquiring data simultaneously in X-, C-, L- and P-bands: an artist's view (X-blue, C-green, L-purple, P-red). To the right, the DO228 with F-SAR installed approaching Gran Canaria airport on the return from the AfriSAR mission in Gabon. Radar antennas visible behind the wing and under the cabin.

\subsection{F-SAR System Design}

The F-SAR system is basically composed of a system control and data acquisition subsystem to which individual RF modules are connected. System control is based on a mixed Extended CAN bus and Ethernet concept. This gives the necessary flexibility and the degrees of freedom to configure the system optimally for carrying out the desired measurements and experiments like bi-static SAR, for instance. Further, the concept makes the extension to any other RF band an easier task (see Fig. 2).

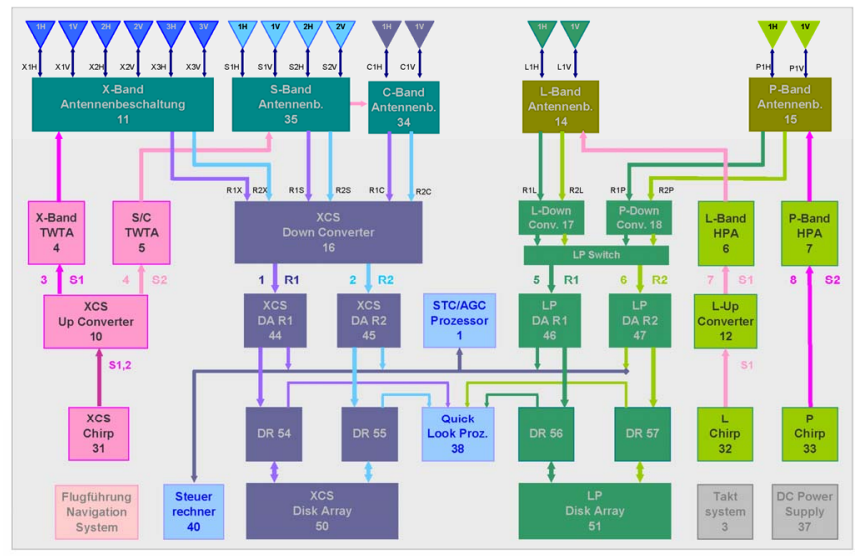

Figure 2. F-SAR system configuration for multifrequency and polarimetric operation in X-C-SL-P-bands including single-pass interferometric capabilities in X-and S-bands. 
A special F-SAR antenna carrier (see Fig. 3 (bottom, centre)) was developed and certified airworthy which carries up to seven planar dual-polarization array antennas: three in X-band, one in C-band, two in S-band and one in L-band. The P-band antenna is mounted under the front part of the cabin as indicated in Fig. 3 (bottom, right).
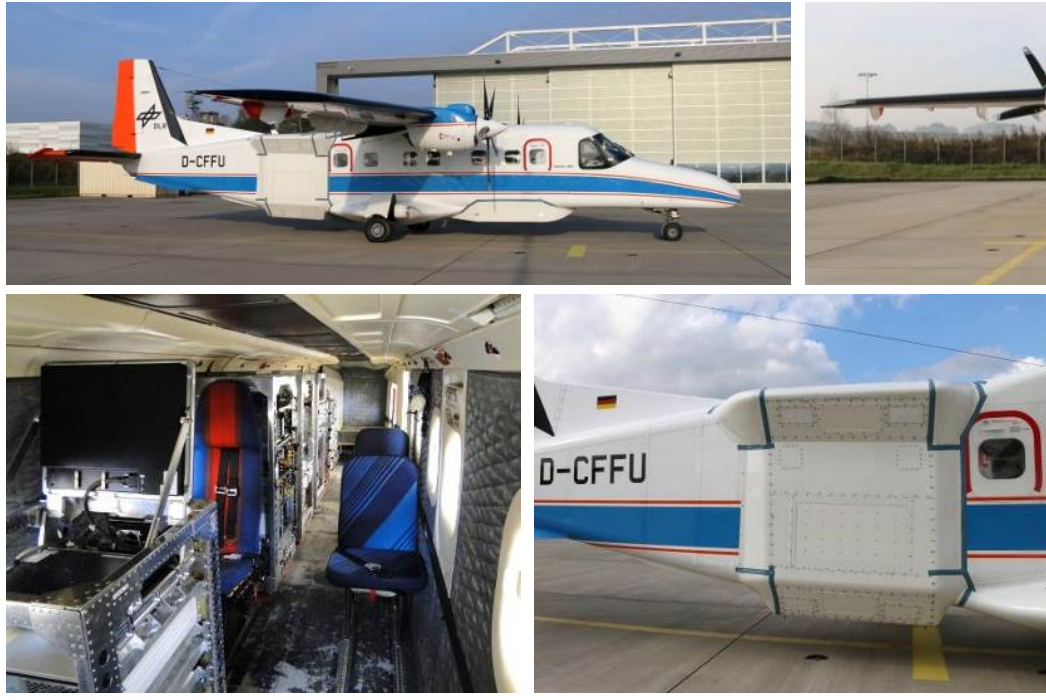

Figure 3. Actual photographs of the DLR DO228 D-CFFU research aircraft with the F-SAR installed on board. Top left: side view; top right: front view; bottom left: a view inside the cabin; bottom centre: F-SAR antenna carrier, close-up view; bottom right: P-band antenna and windshield, close-up view.

The antenna carrier has the important advantage that it makes it easy to change the antenna configuration or to mount other antennas avoiding or keeping at minimum level individual airworthiness certification procedures the same time.

The nominal antenna configuration provides three single-pass interferometers: across track (XTI) in S-band and X-band, and along track (ATI) in X-band. The mechanical baselines are approx. $1.60 \mathrm{~m}(\mathrm{XTI})$ and approx. $85 \mathrm{~cm}$ (ATI).

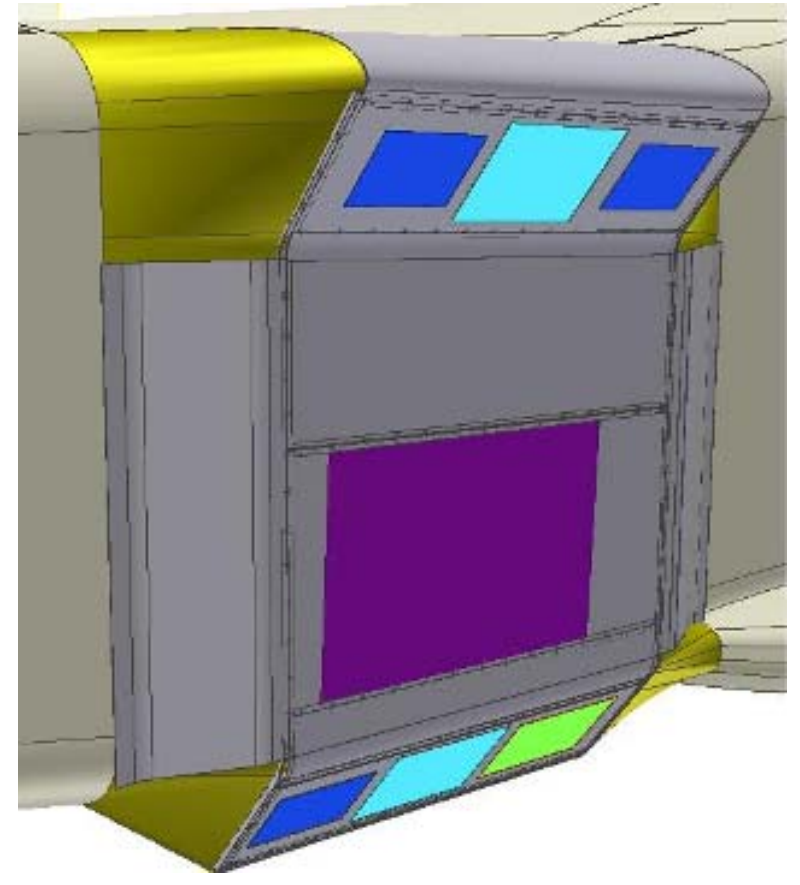

Figure 4. Schematic drawing of the F-SAR antenna mount with the nominal antenna configuration: $3 \mathrm{X}$ band (blue), 2 S-band (light-blue), C-band (light-green) and L-band (purple). 
A central computer unit controls the radar via CAN bus and Ethernet. The required synchronous timing and clock signals are generated in the main timing unit with less than 6ps jitter and rise times of less than $80 \mathrm{ps}$. A $50 \mathrm{MHz}$ ultra-stable Quartz oscillator is the clock reference. A GNSS/IMU based precision navigation system delivers a 1PPS signal which regularly triggers an absolute time stamp in the raw data header.

F-SAR operates with four 1GS-ADCs. Each ADC unit has raw data formatting integrated. High speed data recording units are connected via optical fibre. All data is recorded on solidstate-disks (SSD). They are highly reliable in the operating environment of a turboprop engine aircraft even at flight altitudes well above $3000 \mathrm{~m}$.

\subsection{F-SAR Operational Instrument Configurations}

The DO228-212 aircraft has an empty weight of 3976kg, a maximum zero fuel weight of $5400 \mathrm{~kg}$ and a maximum take-off weight of $6575 \mathrm{~kg}$. The delta of $1424 \mathrm{~kg}$ between zero fuel weight and empty weight is the maximum payload possible on board the aircraft. It is composed of (1) instrument, (2) aircrew and equipment and (3) safety equipment. The difference between take-off weight and zero fuel weight equals the amount of (4) fuel in $\mathrm{kg}$ at departure. With maximum payload this is $2590 \mathrm{lbs}$ of fuel, which is good for about three hours of flight. The DO228 consumes typically 600lbs of JET-A1 fuel per hour. An IFR fuel reserve of $600 \mathrm{lbs}(1 \mathrm{~h})$ is mandatory in regions like Europe. It is $900 \mathrm{lbs}(1.5 \mathrm{~h})$ in remote regions, like the arctic (Greenland, Svalbard), where alternate airports are sparse. The aircrew consists of two pilots, one aircraft engineer and two radar engineers. Each person is counted $80 \mathrm{~kg}$. Based on these numbers just a few F-SAR instrument configurations have been certified airworthy so far. They are summarised in Tab. 2.

Table 2. F-SAR instrument configurations and endurance $\left({ }^{*}\right)$ Configuration not certified up to date.).

\begin{tabular}{lllllll}
\hline Configuration & $\mathbf{X}$ & $\mathbf{C}$ & $\mathbf{S}$ & $\mathbf{L}$ & $\mathbf{P}$ & Endurance \\
\hline F-SAR X-C-S-L & 9.600 & 5.300 & 3.250 & 1.325 & --- & $3.75-4.25 \mathrm{~h}$ \\
F-SAR P & --- & --- & --- & --- & 0.435 & $4.0-4.5 \mathrm{~h}$ \\
F-SAR L & --- & --- & --- & 1.325 & --- & $4.25-4.75 \mathrm{~h}$ \\
F-SAR L-P & --- & --- & --- & 1.325 & 0.435 & $3.5-4.0 \mathrm{~h}$ \\
$\left.F-S A R ~ X-C-S-L-P^{*}\right)$ & 9.600 & 5.300 & 3.250 & 1.325 & 0.435 & $2.75-3.25 \mathrm{~h}$ \\
TERENO Mobile Platform & \multicolumn{2}{l}{ Combination of F-SAR L plus PLMR-2 plus Thermal Cam } & $4.0-4.5 \mathrm{~h}$ \\
\hline
\end{tabular}

F-SAR X-C-S-L configuration is most heavily used. It allows simultaneous data collection in $\mathrm{X}-\mathrm{C}-\mathrm{L}$ or X-S-L modes in a pass. C- and S-bands must be switched from pass to pass for technical reasons. The X-C-S-L configuration offers single $(X, S)$ and repeat (all) pass polarimetric interferometric measurement modes. An extension towards C-band single polarisation $(\mathrm{H}$ or $\mathrm{V})$, single pass interferometry is currently being implemented.

The single frequency configurations F-SAR P and F-SAR L are employed rarely. It is more efficient to combine with shorter wavelengths or other instruments, like the PLMR-2 radiometer and a thermal camera (see TERENO Mobile Platform [2]).

F-SAR L-P configuration is useful for forestry applications to measure biomass for instance, when auxiliary data products, like DEMs, are available from other sources, such as TanDEM$\mathrm{X}$. Measurement modes are TomoSAR and repeat pass Pol-InSAR.

In X-C-S-L-P configuration F-SAR reaches the zero fuel weight limit. This configuration has limited usability because of low endurance and operational restrictions. 


\section{F-SAR Instrument Performance}

Environmental research work of today has tough precision and stability requirements on information collected by modern SAR instruments. This necessitates regular overflights of a calibration test site, precise internal instrument calibration and precise in-door antenna measurements [3]. All supplemented and supported by a highly sophisticated SAR processing facility.

The Microwaves and Radar Institute maintains a test site for external SAR instrument calibration at the former military airfield of Kaufbeuren in the South of Germany (see Fig. 5). This area has 14 conventional trihedral and dihedral corner reflectors of different size ranging from $90 \mathrm{~cm}$ to $250 \mathrm{~cm}$ leg length installed in total. The standard look direction is to the North for all reflectors. An off-nadir angle range from approx. $25^{\circ}$ to $60^{\circ}$ is covered. The site is used for all F-SAR frequency bands, with limitations also for P-band.

We usually check the following system performance parameters and more every time that FSAR is installed on board the aircraft.

- Range and azimuth position

- Range and azimuth resolution

- Radar cross section

- Polarimetric phase

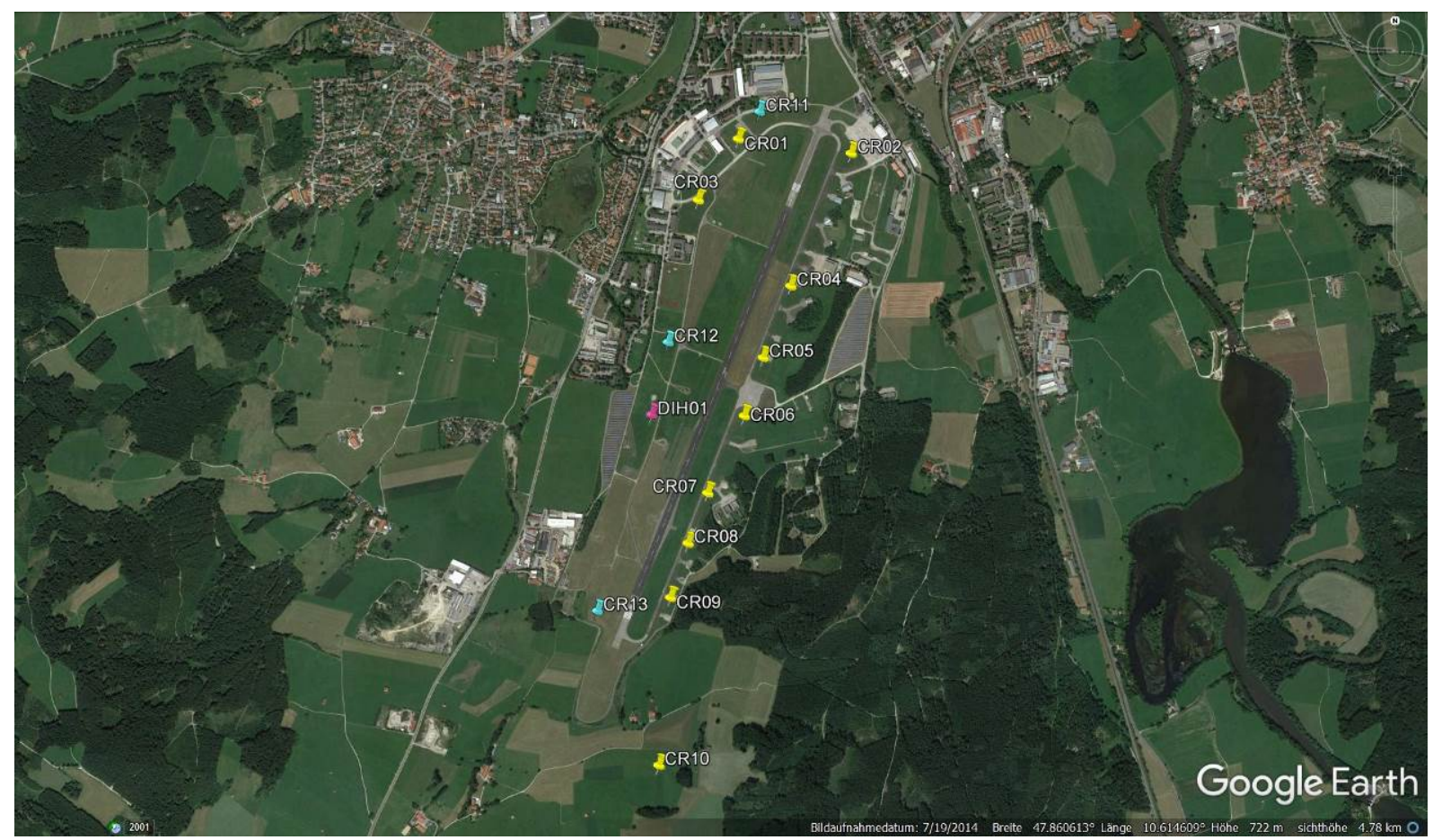

Figure 5. The F-SAR calibration test site 'Kaufbeuren'. Radar reflectors are set up along the runway. Pins indicate their approximate positions.

The Institute operates a Compact Test Range (CTR) for in-door antenna measurements [4]. As part of the external instrument calibration procedure we measure the complex transfer function of each SAR antenna over operational frequency range, alpha and epsilon angles in its immediate surroundings. Meaning, we include antenna carrier and windshield in the measurements. For instance, in Figure 6 the P-band antenna characteristics at centre frequency and horizontal polarisation are shown. 

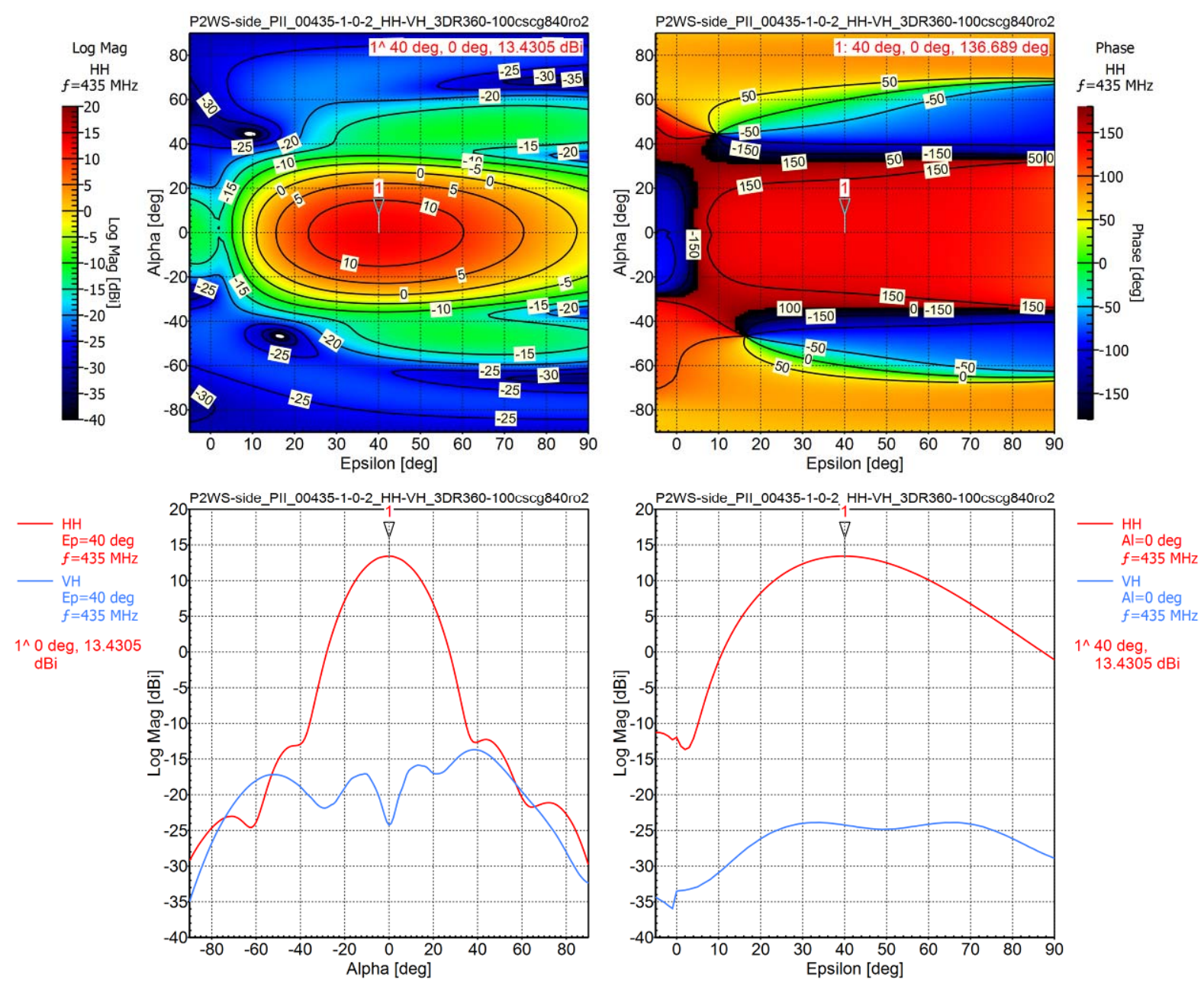

Figure 6. The F-SAR P-band antenna characteristics at $\mathrm{f}_{\mathrm{c}}=435 \mathrm{MHz}$ measured in the Compact Test Range of DLR. Top: Magnitude and phase pattern over alpha and epsilon angles. Bottom: Standard main beam cuts. Cross polarisation level is shown in blue.

During every F-SAR measurement flight we record radar signal replicas before and after each measurement as part of the internal calibration procedure. A replica contains all instrument internal signal transmission errors, i.e. errors in the transmitter and in the receiver chains. Evaluating replicas also helps to monitor instrument stability.

A summary of F-SAR's instrument performance is given Tab. 3 below. These parameters were determined based on raw data collected in 2016, for P-band more specifically data of the AfriSAR campaign.

Table 3. F-SAR instrument performance summary.

\begin{tabular}{|c|c|c|c|c|c|c|c|c|c|}
\hline \multirow[b]{2}{*}{ RF } & \multicolumn{2}{|c|}{ Rg pos error } & \multicolumn{2}{|c|}{ Az pos error } & \multicolumn{2}{|c|}{ RCS error } & \multicolumn{2}{|c|}{ HH/VV phase diff. } & \multirow{2}{*}{$\begin{array}{l}\text { NESZ } \\
\left(25^{\circ} / 45^{\circ} / 60^{\circ}\right) \\
{[\mathrm{dB}]}\end{array}$} \\
\hline & $\begin{array}{l}\mu_{\mathrm{rg}} \\
{[\mathrm{m}]}\end{array}$ & $\begin{array}{l}\sigma_{\mathrm{rg}} \\
{[\mathrm{m}]}\end{array}$ & $\begin{array}{l}\boldsymbol{\mu}_{\mathrm{az}} \\
{[\mathbf{m}]}\end{array}$ & $\begin{array}{l}\sigma_{\mathrm{az}} \\
{[\mathrm{m}]}\end{array}$ & $\begin{array}{l}\boldsymbol{\mu} \\
{[\mathrm{dB}]}\end{array}$ & $\begin{array}{l}\sigma \\
{[\mathrm{dB}]}\end{array}$ & & $\begin{array}{l}\sigma \\
{\left[^{\circ}\right]}\end{array}$ & \\
\hline $\mathbf{X}$ & -0.005 & 0.045 & 0.013 & 0.052 & -0.152 & 0.275 & 1.09 & 4.12 & $-32 /-34 /-28$ \\
\hline $\mathbf{C}$ & -0.005 & 0.045 & 0.013 & 0.052 & 0.058 & 0.396 & 0.581 & 1.36 & $-33 /-40 /-27$ \\
\hline $\mathbf{S}$ & 0.007 & 0.051 & 0.034 & 0.062 & -0.133 & 0.338 & 0.254 & 2.08 & $-28 /-34 /-30$ \\
\hline $\mathbf{L}$ & 0.008 & 0.048 & -0.024 & 0.035 & 0.125 & 0.184 & 1.07 & 1.67 & $-40 /-46 /-42$ \\
\hline $\mathbf{P}$ & -0.064 & 0.095 & -0.016 & 0.078 & -0.139 & 0.268 & 0.188 & 2.98 & $-44 /-46 /-46$ \\
\hline
\end{tabular}


As an F-SAR image example a polarimetric C-band look of the calibration site Kaufbeuren is given in Figure 7. The image is geo-coded using a terrain model in UTM32/WGS84.

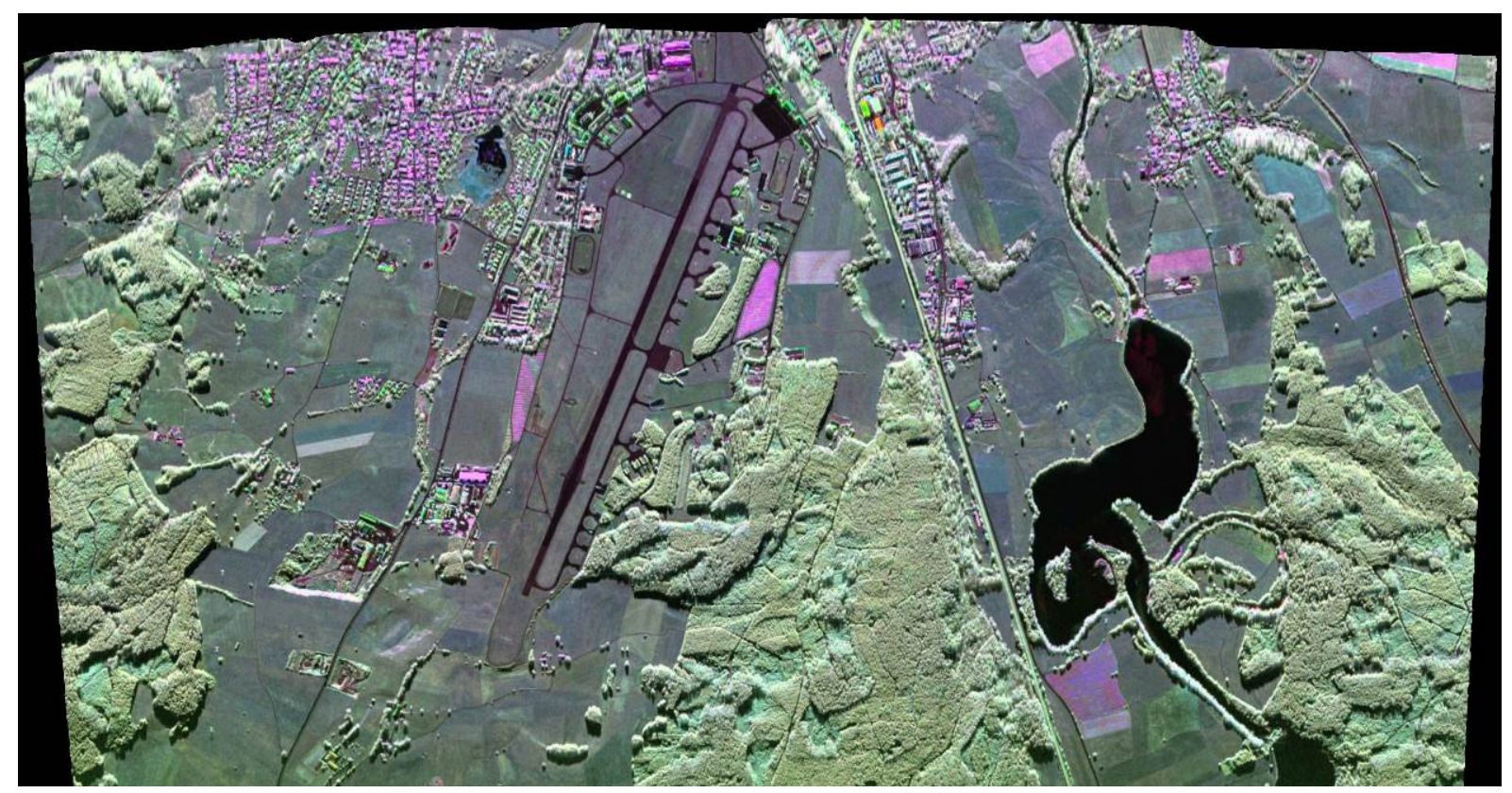

Figure 7: F-SAR polarimetric C-Band image of the calibration site Kaufbeuren. Altitude of flight is $3048 \mathrm{~m}$ above WGS84 ellipsoid or approx. 2300m above average ground level.

\section{Prominent Campaign Activities}

In the past two years two very prominent F-SAR campaigns were carried out. The first one in spring 2015 led to Greenland, where the performance of the radar after the completion of system updates (new P-band radar segment) was tested and an enormous amount of data on snow and ice collected in cooperation with the Danish Defence Acquisition and Logistics Organization (DALO) [5]. The second in winter 2016 took place in Gabon in cooperation with ESA, ONERA, NASA and the Gabonese national space agency AGEOS focusing on African tropical rainforest [6].

\subsection{The ARCTIC 2015 Campaign}

Principal goal of this campaign was to demonstrate the capabilities of SAR for security applications in arctic environments, as well as to investigate various advanced methods for extracting ice and snow parameters from SAR data. During this campaign, the ability of FSAR to simultaneously record fully-polarimetric SAR data in several frequency bands was used for the first time on large scale. It was also the first campaign the new P-band segment was employed for radar measurements.

After a lead time of about nine months required for all preparations the ARCTIC campaign started on April 27, 2015 with a calibration test flight over the town Kangerlussuaq. It followed 23 more research flights over several test sites distributed south of latitude $70^{\circ} \mathrm{N}$ along the Greenlandic coast, on the ice shield and the Davies straight (see Figure 8). A team of three scientists had equipped four sites (DYE-3, South Dome, EGIG and Helheim) with radar reflectors just before campaign start, an adventurous undertaking. The final flight was conducted on May 24, 2015. 


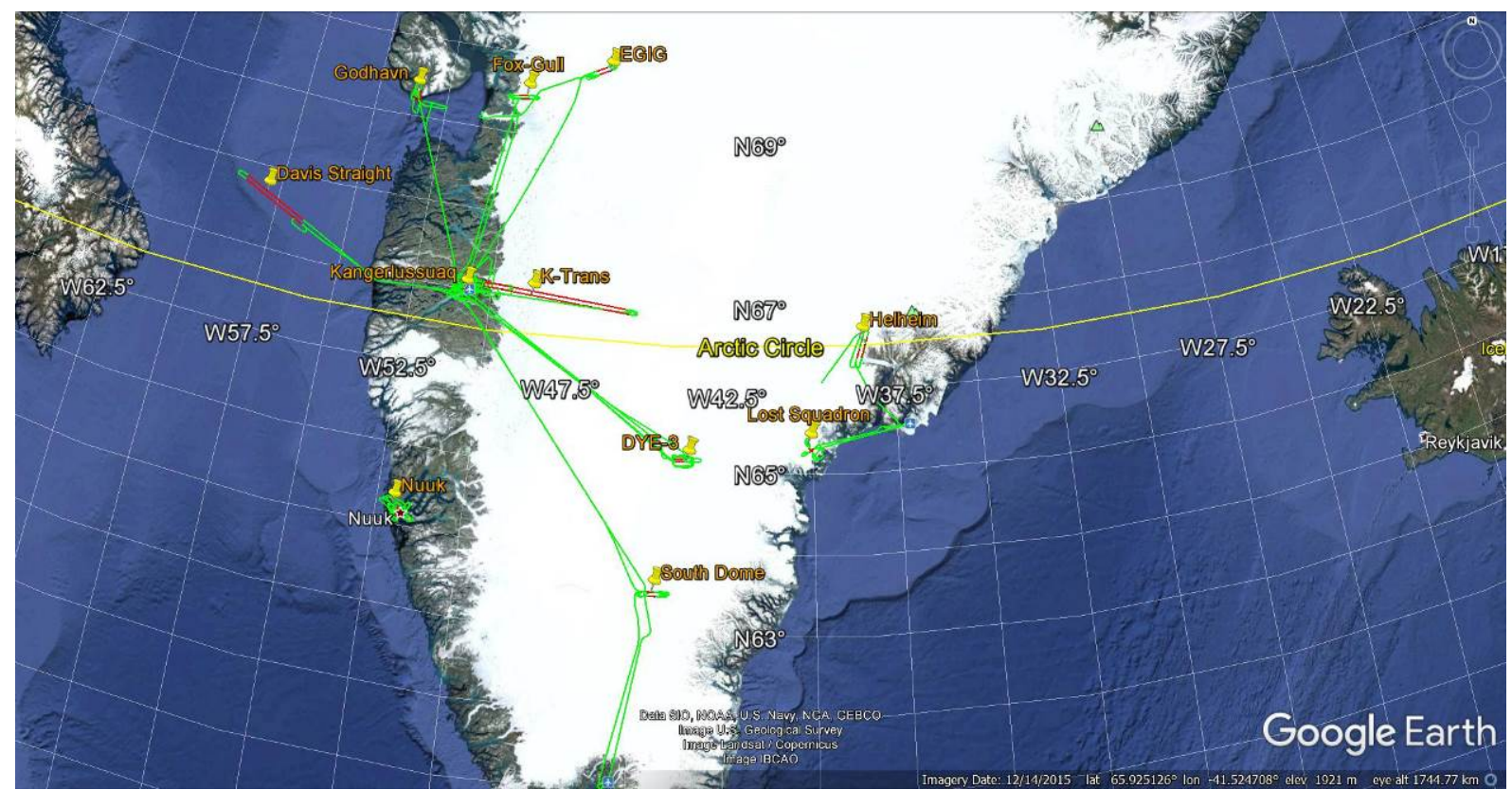

Figure 8: ARCTIC 2015 test site locations spread over Southern Greenland and Davis Straight.

Primary mission base was Kangerlussuaq airport. From here most of the measurement flights were conducted. In a few cases transfer flights to the secondary bases Kulusuk, Nuuk, Narsarsuaq and Illuisat were required prior to measurements. F-SAR image examples are shown below in Figures 9 and 10.
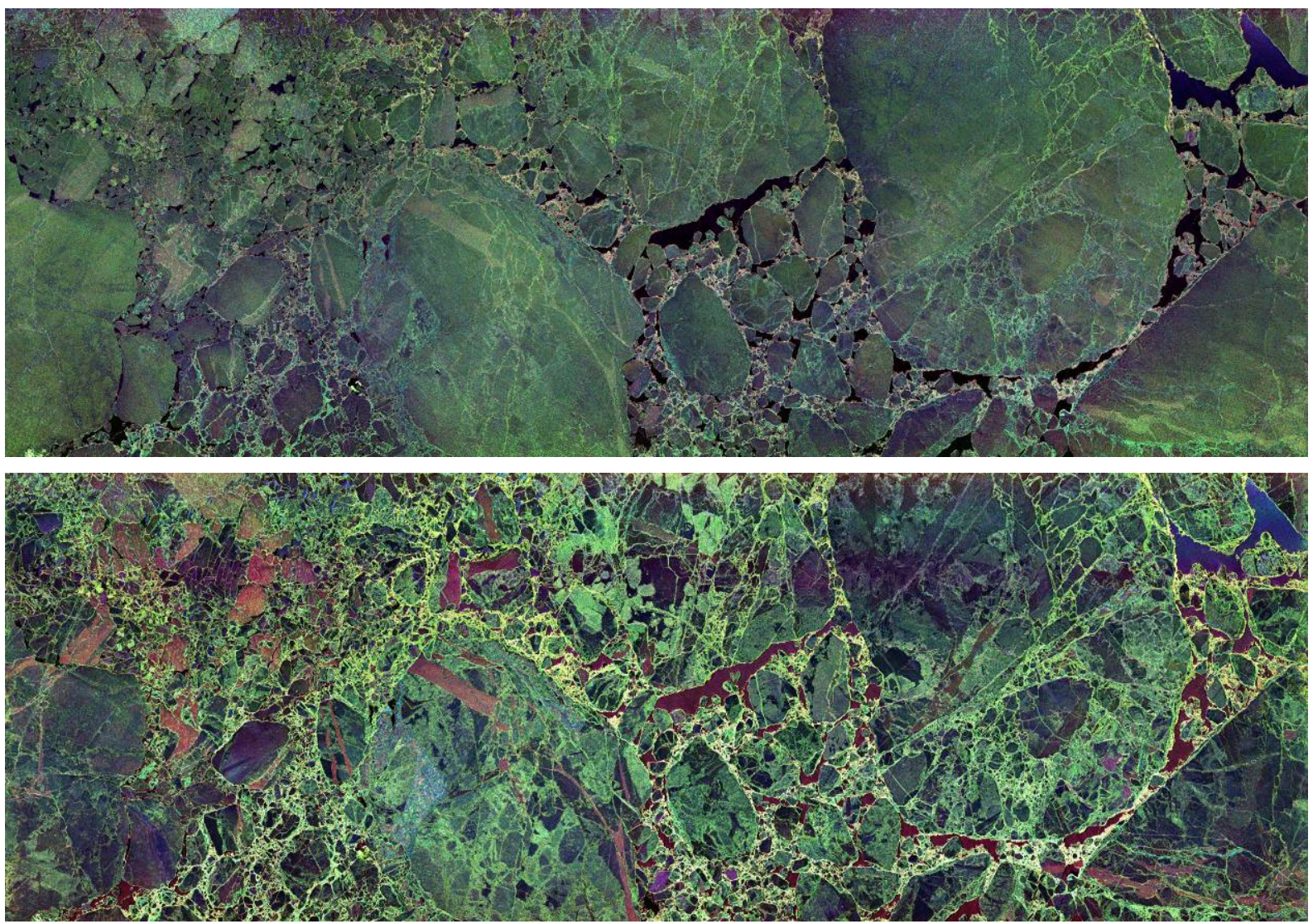

Figure 9: F-SAR polarimetric images of sea ice in the Davis Straight. Top: X-band. Bottom: S-band. Both scenes were simultaneously recorded in X-S-L mode. They show exactly the same area in the arctic ocean. 
The radar's ability to record multi-spectral data showed fascinating results especially over ablation areas of the Greenlandic ice sheet.
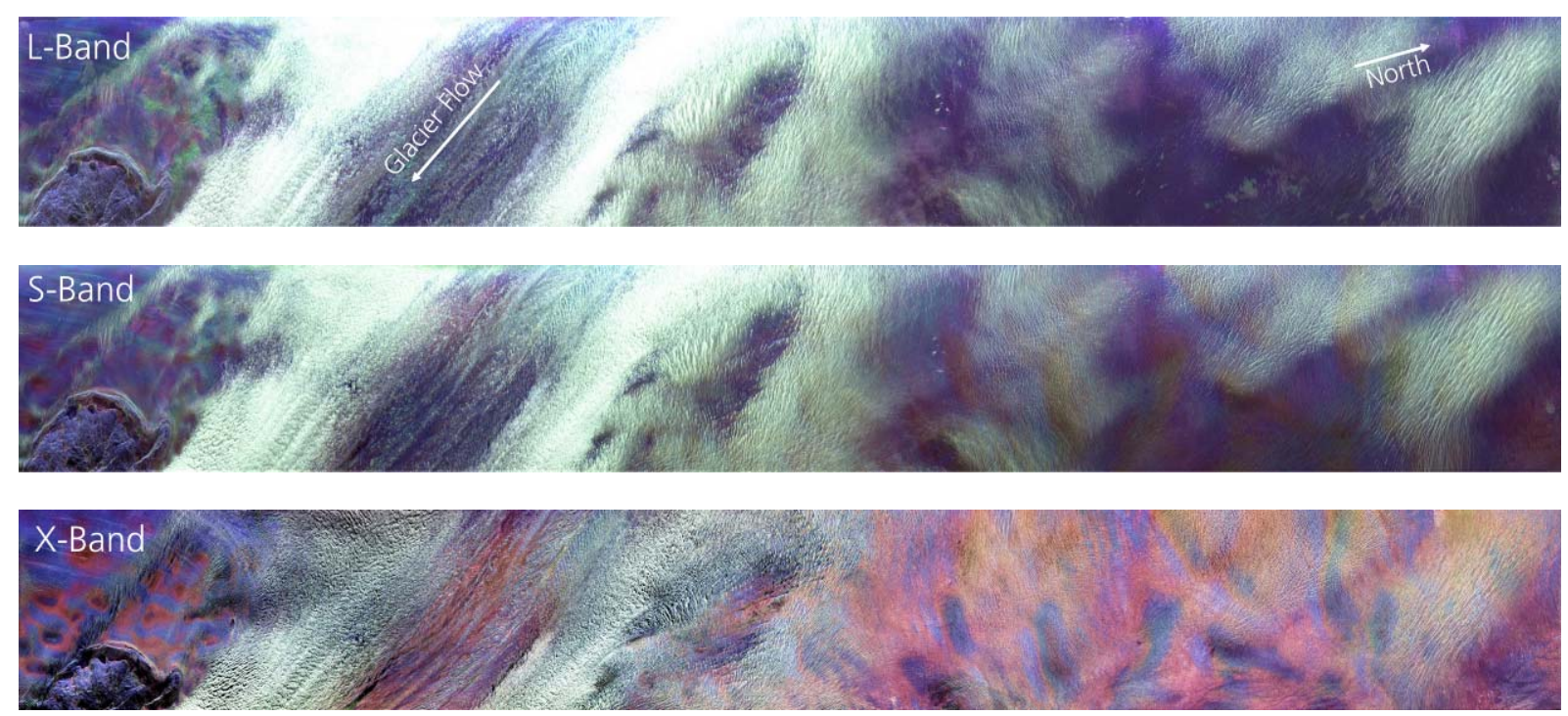

Figure 10: Helheim Glacier, polarimetric images recorded simultaneously in L-, S- and X- bands. Pauli decomposition R, G, B = HH-VV, HV, HH+VV.

\subsection{The AfriSAR 2016 Campaign}

The AfriSAR campaign was designed and executed to support development and assessment activities in the frame of ESA's forthcoming BIOMASS P-band SAR satellite mission. The launch is envisaged for 2020.

The campaign was split in two phases, the first in June/July 2015 covered by ONERA, the second in February 2016 by DLR with the F-SAR system in the new L-P-band dual-frequency system configuration. This opportunity was used by DLR to collect not only P-band data but perform SAR measurements in L-band in parallel in support of the proposed German Tandem-L spaceborne SAR mission. Figure 11 shows the test site locations in Gabon.

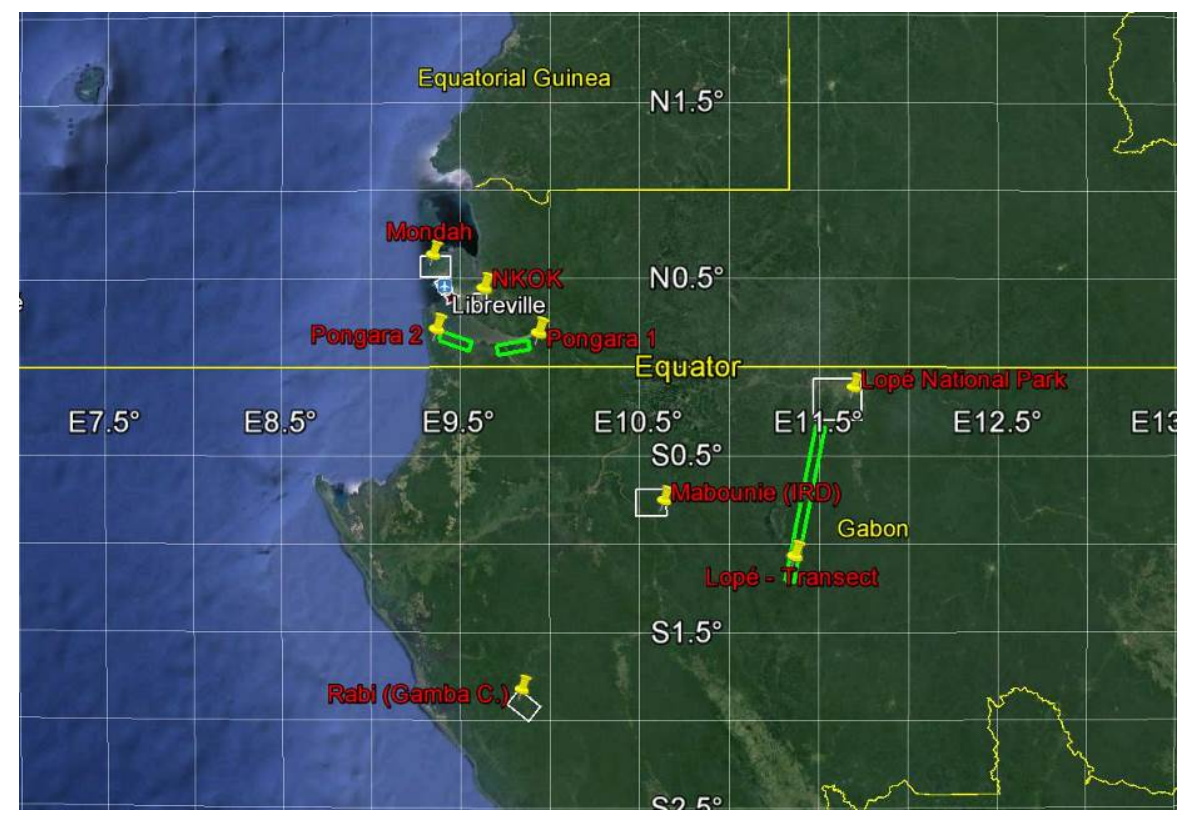

Figure 11: AfriSAR 2016 test site locations spread over Gabon. All flights were conducted out of the mission base, Libreville Airport. Distances:

La Lopé: $250 \mathrm{~km}$

Mabounie: $180 \mathrm{~km}$

Rabi: $260 \mathrm{~km}$

Mondah: $15 \mathrm{~km}$

Nkok: $24 \mathrm{~km}$

Pongara: $40 \mathrm{~km}$ 
Figure 12 shows a selection of L- and P-band images of the Pongara mangroves on the southern banks of the Gabon estuary.
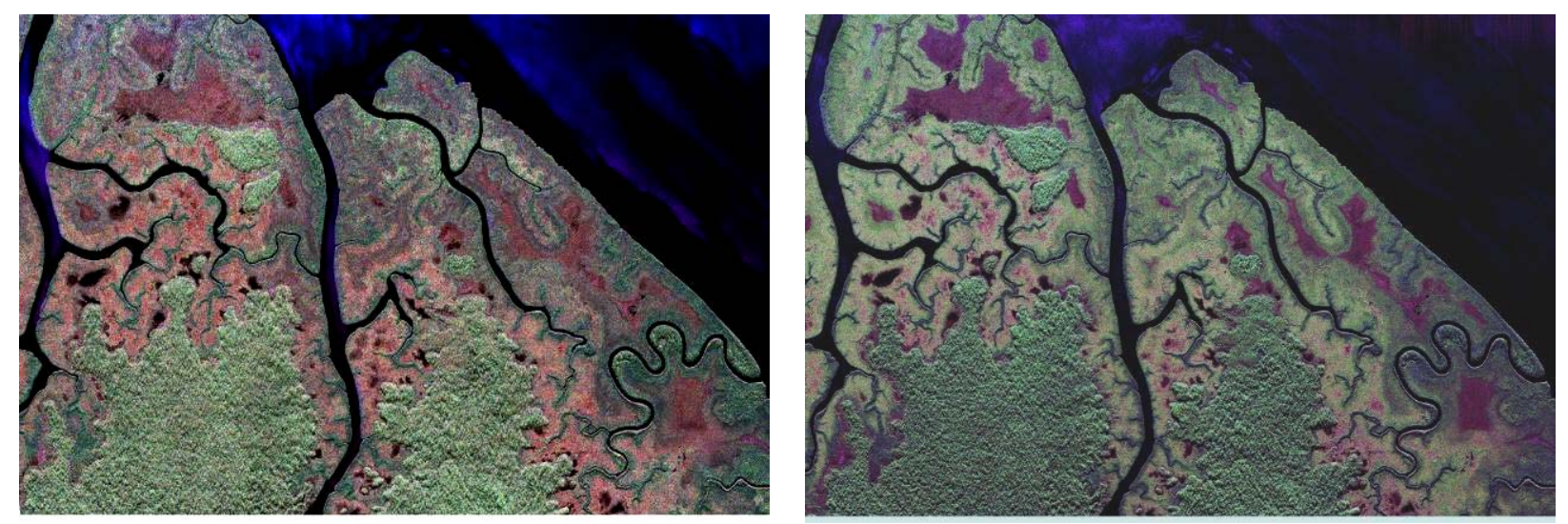

Figure 12: F-SAR image examples of Pongara-2 test site. Cut-out of larger scene. P-band (left) and L-band (right), recorded simultaneously.

\section{Summary}

Over the past couple of years F-SAR has evolved to a versatile and reliable multi-frequency SAR measurement instrument. This has been achieved by careful system design, concentrated work on data processing and calibration as well as specific improvements in antenna measurement. All five frequency bands can now be made available to researchers.

\section{References}

[1] A. Reigber, R. Scheiber, M. Jäger, P. Prats, I. Hajnsek, T. Jagdhuber, K.P. Papathanassiou, M. Nannini, E. Aguilera, S. Baumgartner, R. Horn, A. Nottensteiner, A. Moreira, "Very-HighResolution Airborne Synthetic Aperture Radar Imaging: Signal Processing and Applications", Proceedings of the IEEE, Vol. 101(3), pp. 759-783, March 2013.

[2] Carsten Montzka, Thomas Jagdhuber, Ralf Horn, Heye R. Bogena, Irena Hajnsek, Andreas Reigber, and Harry Vereecken, "Investigation of SMAP fusion algorithms with airborne active and passive L-band microwave remote sensing", IEEE TGRS, Vol. 54(7), pp. 38783889, July 2016.

[3] M. Jäger, A. Reigber, and R. Scheiber, "Accurate consideration of sensor parameters in the calibration and focusing of F-SAR data", Proceedings of the European Conference on Synthetic Aperture Radar, Nürnberg, Germany, pp. 20-23, April 2012.

[4] Limbach, M., Gabler, B., Di Maria, A., Horn, R. and Reigber, A., "DLR-Compact Test Range Facility", European Conference on Antennas and Propagation (EuCAP), Prague, Czech Republic, 2012. ISBN 978-1-4577-0918-0.

[5] Andreas Reigber, Ernst Krogager, Martin Keller, Marc Jäger, Irena Hajnsek, Ralf Horn, "The DALO-ARCTIC campaign: Multi-spectral SAR imaging of Ice Features in Greenland", Proceedings of the $11^{\text {th }}$ European Conference on Synthetic Aperture Radar, Hamburg, Germany, pp. 293-295, June 2016.

[6] "Technical Assistance for the Development of Airborne SAR and Geophysical Measurements during the AfriSAR Experiment", ESA study contract final report, contract no. 4000114293/15/NL/CT, 2016. 